\title{
Clinical investigation of lesions of the visual pathway: a new objective technique
}

\author{
D. REGAN AND J. R. HERON \\ From the Department of Communication, University of Keele, and the Department of Neurology, \\ North Staffordshire Royal Infirmary
}

There have been several previous reports of the use of the gross electrical brain potentials evoked by visual stimulation (evoked potentials) to provide objective indications in visual disorders (Vaughan, Katzman, and Taylor, 1963; Copenhaver and Beinhocker, 1963; Vaughan and Katzman, 1964; Potts and Nagaya, 1965; Nagata and Jacobson, 1966; Balen, Gon, and Hellendoorn, 1966; Schreinemachers and Henkes, 1968; Jacobson, Hirose, and Suzuki, 1968). A popular method has been to direct brief, very bright, flashes of light into one or both eyes, and to extract the scalp evoked potentials from the spontaneous EEG by computer averaging. The potentials evoked in this way are not easily quantified, and it is therefore difficult to define clearly the difference between 'normal' and 'abnormal' responses. It is also difficult to compare the integrities of foveal and extrafoveal pathways. The notorious variability of evoked potentials imposes a severe limitation on the precision with which relationships may rapidly be measured between responses to stimulations of different areas of the retina, when such stimulations are performed successively (Copenhaver and Beinhocker, 1963; Potts and Nagaya, 1965; Nagata and Jacobson, 1966; Balen et al., 1966; Jacobson et al., 1968; Schreinemachers and Henkes, 1968).

This is a preliminary report of a new method of investigating visual field defects which is objective, fairly rapid, non-traumatic, and makes comparatively small demands on the patient. We have developed a method which enables two areas of the retina to be stimulated simultaneously and weakly. The results of this are to minimize the effect of evoked potential variability, to minimize the effect of light scattered within the eye, and to improve patient cooperation. Our method gives results in both visual and quantitative forms, and can distinguish between different parts of the visual field by using different kinds of stimuli.

\section{METHODS}

The nasal and temporal half-fields of one eye were simultaneously stimulated by weakly flickering light, or by chess-board patterns whose black and white squares alternated. The repetition frequencies of the stimuli falling on the left and right half-fields were sufficiently similar to be effectively identical for the evoked potential system, but sufficiently different for the analysing equipment to isolate and measure the individual responses to left and right half-field stimulation (Regan and Cartwright, in press). A stable difference between the left and right stimulation frequencies of about $0.3 \mathrm{c} / \mathrm{s}-$ for example, 18.3 and $18.0 \mathrm{c} / \mathrm{s}$-was found to be suitable. This method of simultaneously recording two signals had the effect of reducing the effect of variability and so enabling the difference between the potentials evoked by left and right half-field stimulation to be measured with precision. In comparison with the method of separately measuring the responses to half-field stimulation, the present technique reduces the effect of variability by a large amount. A reduction of roughly six times is typical, which is equivalent to increasing the speed of the procedure 36-fold. A recording time of about $25 \mathrm{sec}$ has been found adequate to obtain one left and right pair of responses. The analysis of the EEG was carried out by (1) a Fourier Analyser (Regan and Cartwright, in press) (Regan, 1966) and (2) a tape recorder and averaging computer. The analyser possessed the special feature of displaying moment-to-moment responses which could then conveniently be related to changes in the patient's clinical condition.

Two types of stimulator were used. One consisted of two weakly flickering panels whose intensities were sinusoidally modulated to depths of $20 \%$. The panels were separated by a steadily illuminated vertical strip which subtended $1^{\circ}$ at the eye. The patient gazed steadily at a point between the two halves of the stimulator. The use of sinewave modulated intensity has the advantage that distortions in the response waveform can be confidently attributed to the brain (Tweel and Verduyn Lunel, 1965; Regan, 1968a). A further advantage of sinewaves over the widely used brief flashes or square waveforms is that the effective stimulus frequency is known (Tweel and Verduyn Lunel, 1965; Regan, 1968a). The use of low modulation depths may have comparatively little effect on the amplitude of the response (Tweel and Verduyn Lunel, 1965; Spekreijse, 1966; Regan, 1968a), but it does result in more linear behaviour, which can be handled more easily, and, in addition, provides a weak and reasonably inoffensive stimulus. 
The other stimulator was of a completely different kind. The left and right halves were steadily illuminated chessboard patterns. The two patterns rhythmically performed oscillations through one square's width so that the black and white squares appeared to interchange regularly. Again, there was a central, steadily illuminated, $1^{\circ}$ strip, and the patient gazed steadily at a cross at the centre of the strip.

\section{CASE REPORT}

Mrs. P.K., aged 30 years, was admitted to the Neurological Unit of the North Staffordshire Royal Infirmary in October 1968. Three days previously she had suddenly lost the vision in both eyes. This was followed by severe throbbing left hemicrania, occipital headache, vertigo, nausea, and vomiting. She was unsteady in walking. Vision returned after one hour. Headache and vomiting persisted until her admission. On general examination there were no abnormal physical signs. Her blood pressure was $130 / 80 \mathrm{~mm} \mathrm{Hg}$. Nervous system examination showed nystagmus on lateral gaze, impaired pin-prick sensation on the left side of the face circumorally, and a patchy sensory impairment to painful stimuli over the left side of the body. These signs resolved within 24 hours of admission. No other neurological signs were present.

For some years she had experienced sporadic frontal headaches. These had recently increased in duration and severity and lateralized to the left side of the head. They were associated with blurring of vision, vertigo, ataxia, and nausea or vomiting. In some attacks she experienced a feeling of coldness and weakness in the left leg.

Investigations included: haemoglobin $14 \mathrm{~g} / 100 \mathrm{ml}$., white cell count $6,000 / \mathrm{cu}$. mm, normal differential count, ESR $10 \mathrm{~mm}$ in $1 \mathrm{hr}$ (Westergren), urea $30 \mathrm{mg} \%$, calcium $9.5 \mathrm{mg} \%$, WR negative, liver function tests normal, $24 \mathrm{hr}$ urine assays for catecholamines and 5-hydroxyindole acetic acid normal. The ECG was physiological. Serial electroencephalograms (EEGs) showed no lateralizing or localizing abnormality. There was a slight excess of theta rhythms randomly distributed in the records. Lumbar puncture was normal. Radiographs of the chest and skull were normal. A diagnosis of basilar migraine was made and the patient was discharged from hospital.

Three weeks after her discharge she was readmitted with severe frontal and occipital headache, photophobia, nausea, and vomiting. On examination, she had a right homonymous hemianopia, contact dysaesthesia, and patchy sensory impairment to painful stimuli in the right hand and right leg. Tendon reflexes were increased in the right arm and leg. The plantar responses were flexor. A left vertebral angiogram showed occlusion of the left posterior cerebral artery (Fig. 3). Perimetry confirmed a right homonymous hemianopia with macular sparing (Fig. 2). The EEG again showed no focal or lateralizing abnormalities (Fig. 4).

It was accepted that occlusion of the left posterior cerebral artery was a complication of a severe attack of basilar migraine. Since her discharge she has been seen regularly in the Neurological Clinic. Her headaches have been infrequent. The right homonymous hemianopia is persistent. There are no other neurological signs.
RESULTS

We have used our objective method to investigate visual field defects in Mrs. P.K.

Figure 1 shows that steady-state potentials evoked by $18 \mathrm{c} / \mathrm{s}$ sinusoidally modulated light were almost absent when the right visual field was stimulated, while clear responses were simultaneously evoked by stimulation of the left visual field. ${ }^{1}$ In contrast, the potentials evoked by the alternating chess-board stimulus were much more similar for left and right half-fields. Now the alternating chessboard stimulus evokes potentials almost entirely by stimulation of the central $3^{\circ}$ to $4^{\circ}$ macular area of the retina (Spekreijse, 1966), while responses to sinusoidally modulated intensity may have appreciable contributions from non-foveal stimulation (central $20^{\circ}$ to $30^{\circ}$ ) (Regan, 1966; Spekreijse, 1966). The flicker results of Fig. 1 could, therefore, be explained by the presence of a lesion involving the pathway from the right half-field and extending close to the fovea; furthermore, the chess-board results in Fig. 1 could be explained by macular sparing of at least the central $3^{\circ}$ to $4^{\circ}$.

Sutsequent perimetry showed a right homony mous hemianopia with macular sparing (Fig. 2R A left vertebral angiogram showed an occlusion on the left posterior cerebral artery (Fig. 3). The results $\bigcirc$ of the evoked potential method, therefore, agreed with both the perimetry findings and the site of the lesion as determined by vertebral angiography.

\section{DISCUSSION}

We have developed a new objective method for the investigation of visual fields, and applied this method to a patient with basilar migraine. There was clinical evidence of a persistent right hemianopia. Vertebral angiography confirmed that the left posterior cerebral artery was occluded. Subjective perimetry and our objective method were in complete agreement in defining a right hemianopia with macular sparing.

The principle of our method is to compare the gross brain potentials, recorded at the scalp, which are evoked by simultaneous but separate stimulation of two regions of the retina of a single eye. This method provides a 'baseline', in that abnormal

'The assumption that stimulation of a half-field evokes potentials entirely in the corresponding hemisphere may not be justified. Although the effects of light scattered within the eye have been minimized, transcallosal connections may operate. On the other hand, such connections are probably of little importance in this study because a $1^{\circ}$ central vertical retinal strip was unstimulated; in monkey, baboon, and cat, callosal connections relate directly only to areas $c$ close to the vertical meridian (Choudhury, Whitteridge, and Wilson, 1965; Hubel and Wiesel, 1967). Note: For convenience, the potentials evoked by stimulation of the left and right half-fields have been related to the right and left hemispheres in Figure 1. 
ALTERNATING CHESSBOARD

$$
F=3.7 \mathrm{c} / \mathrm{sec}
$$

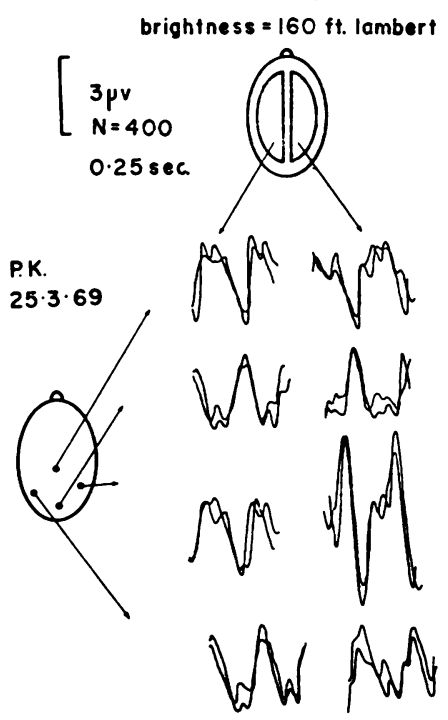

responses which may be evoked by stimulation of part of a visual field can be compared with the responses evoked by simultaneous stimulation of a 'reference' area of the same visual field. So far we have used no more than two simultaneous inputs to one eye. In principle, however, several simultaneous inputs could be used to different parts of the same or both eyes.

Mrs. P.K. was investigated by simultaneously stimulating the left and right half-fields of one eye (right) with weakly modulated lights (weak flicker). Evoked potentials were recorded which were much larger for stimulation of the right than of the left

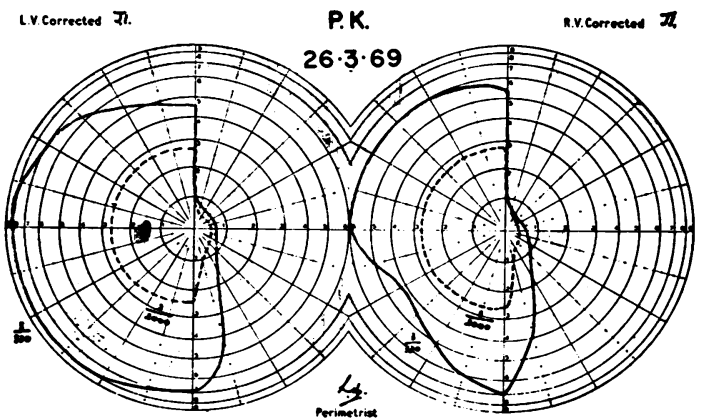

FIG. 2. Perimetry illustrating a right homonymous hemianopia with macular sparing. half of the retina. This suggested a hemianopic field defect on the right side. However, when the left and right halves of the retina were stimulated by altern-

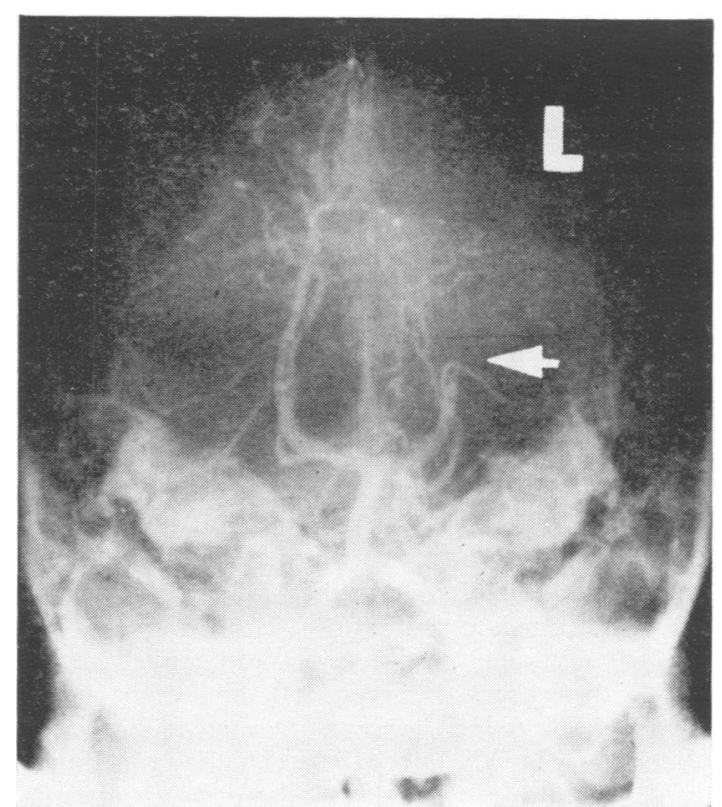

FIG. 3. Vertebral angiogram showing an occlusion of the left posterior cerebral artery.
FIG. 1. Steady-state evoked potentials simultaneously recorded from the left and right hemispheres. Monopolar recordings with left mastoid reference. Right mastoid grounded. Potentials evoked in left and right hemispheres by alternating chess-board are of similar amplitudes for midline electrodes. This indicates macular sparing (greater than $3^{\circ}$ to $4^{\circ}$ ). In contrast, the potentials evoked by sinusoidally modulated flicker in the left hemisphere are much smaller than the potentials evoked in the right hemisphere. This indicates a right-field hemianopia (see footnote on p. 480). 

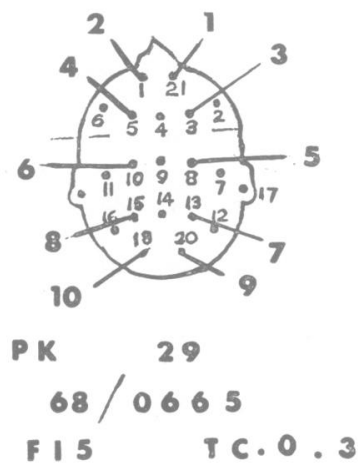

EYES SHUT

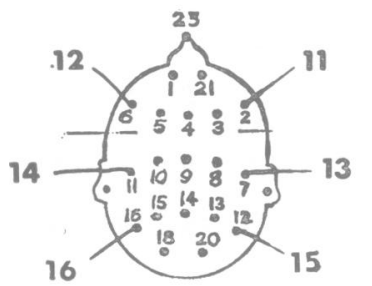

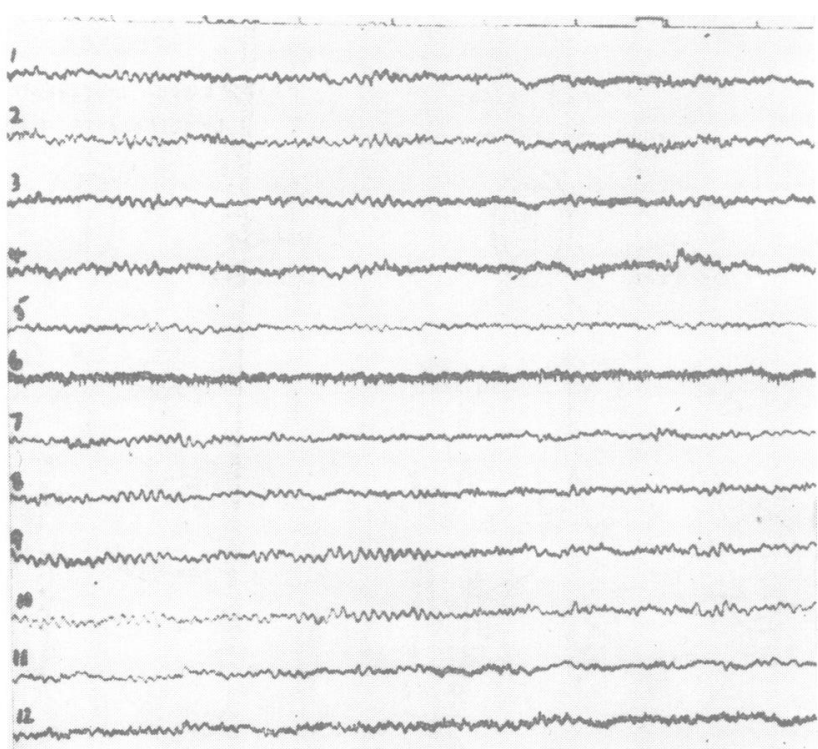

B

14

15

16 ating chess-boards instead of flicker there was a completely different result. The left and right responses now had similar amplitudes. Since the responses to alternating chess-boards are due almost entirely to stimulations of the central $3^{\circ}$ to $4^{\circ}$, this suggests that the left and right halves of the macula gave similar responses. The evoked potential method therefore indicated a right hemianopic field defect with macular sparing. This prediction was later confirmed by perimetry.

Our method has the following features. Simultaneous recordings can be made at a single scalp electrode in a variety of positions of the gross electrical brain responses to monocular stimulation of the individual left and right half-fields. The effect of variability is considerably reduced by recording the left and right responses simultaneously, so that small differences between left and right can be detected. By using different types of visual stimuli a distinction can be made between the contributions of fovea and extra-fovea to the evoked potentials. Recording sessions are short as a result of using rapidly repeated stimuli. This has the effect of encouraging patient co-operation, and also leads to more reliable results. The quantitative results which are made available by the Fourier Analyser express unambiguously the differences between left and right responses. There is evidence that changes in stimulus intensity and in stimulus pattern excite in the brain populations of neurones, which differ functionally, and possibly also anatomically (Spekreijse, 1966; MacKay, 1969; Regan and Heron, 1969). There is also evidence that different populations of neurones are excited by visual stimuli whose intensities are modulated at frequencies of roughly $10 \mathrm{c} / \mathrm{s}, 16 \mathrm{c} / \mathrm{s}$, and $53 \mathrm{c} / \mathrm{s}$ respectively (Tweel and Verduyn Lunel, 1965; Spekreijse, 1966; Regan, $1968 \mathrm{~b}, \mathrm{c})$. These last points suggest the possibility of attempting to locate brain lesions by recording scalp potentials evoked by different kinds of stimuli modulated at different frequencies. Our method may, therefore, have wider applications in clinical neurology than is indicated in this present communication.

In a parallel study, our method has been applied in migraine to measuring the left/right differences 
between and during attacks (Regan and Heron, 1969). The ratio between the potentials evoked by simultaneous stimulation of the left and right halffields of one eye may change considerably $(200 \%$ to $500 \%$ ) during a migraine attack. The application of our method to the non-traumatic location of neurological lesions which affect the visual pathways is also being explored.

\section{SUMMARY}

The method of simultaneous recording of the scalp potentials evoked by visual stimulation of the left and right half-fields of one eye was applied to a patient with homonymous hemianopia and macular sparing. The method predicted this visual field defect and this was confirmed by perimetry. It is suggested that in addition to its application to visual field disorders this technique has wider applications to the localization of cerebral lesions.

We are grateful to the Medical Research Council, the Migraine Trust, and Sandoz Pharmaceuticals for support. It is a pleasure to thank R. F. Cartwright for technical and design assistance. Our thanks are also due to $\mathrm{Mr}$. $\mathrm{H}$. Wardell, Mr. R. Morrell, and Mr. H. Slynn of the Keele University Workshop for their valuable support.

\section{REFERENCES}

Balen, A.Th. van, Gon, J. J. Denier van der, and Hellendoorn, E. H. (1966). The differentiation between responses of foveal and extrafoveal stimuli in the E.R.G. and E.E.G. Vision Res. Suppl., 255-262.

Choudhury, B. P., Whitteridge, D., and Wilson, M. E. (1965). The function of the callosal connections of the visual cortex. Quart. J. exp. Physiol., 50, 214-219.
Copenhaver, R. M., and Beinhocker, G. D. (1963). Evoked occipital potentials recorded from scalp electrodes in response to focal visual illumination. Invest. Ophthal., 2, 393-406.

Hubel, D. H., and Wiesel, T. N. (1967). Cortical and callosal connections concerned with the vertical meridian of visual fields in the cat. J. Neurophysiol., 30, 1561-1573.

Jacobson, J. H., Hirose, T., and Suzuki, T. A. (1968). Simultaneous E.R.G. and V.E.R. in lesions of the optic pathway. Invest. Ophthal., 7, 279-292.

MacKay, D. M. (ed.) (1969). Evoked potentials as indicators of sensory information processing. M.I.T. Neurosci. Res. Program Bull. (In press.)

Nagata, M., and Jacobson, J. H. (1966). Combined E.R.G. and occipital response recording. Clinical Electroencephalography, Vision Res. Suppl., 249-255.

Potts, A. M., and Nagaya, T. (1965). Studies on the visual evoked response. Invest. Ophthal., 4, 303-309.

Regan, D., and Cartwright, R. F. (1969). A method of measuring the potentials evoked by simultaneous stimulation of different retinal regions. Electroenceph. Clin. Neurophysiol. (In press.)

- (1966). Some characteristics of average steady-state and transient responses evoked by modulated light. Electroenceph. clin. Neurophysiol., 20, 238-248.

- (1968a). Evoked potentials and sensation. Perception and Psychophysics., 4, 347-350.

evoked potentials from the left and right hemispheres in migraine, in Background to Migraine. Heinemann: London. (In press.)

(1968b). Chromatic adaptation and steady-state evoked potentials. Vision Res., 8, 149-158.

- (1968c). A high frequency mechanism which underlies visual evoked potentials. Electroenceph. clin. Neurophysiol., 25, 231237.

Schreinemachers, H. P., and Henkes, H. E. (1968). Relation between localized retinal stimuli and the visual evoked response in man. Ophthalmologica (Basel), 155, 17-27.

Spekreijse, H. (1966). Analysis of E.E.G. Responses in Man; evoked by Sine Wave Modulated Light. Thesis, University of Amsterdam. Junk Publishers: The Hague.

Tweel, L. H. van der, and Verduyn Lunel, H. F. E. (1965). Human visual responses to sinusoidally modulated light. Electroenceph. clin. Neurophysiol., 18, 587-598.

Vaughan, H. G., Jr., and Katzman, R. (1964). Evoked response in visual disorders. Ann. N.Y. Acad. Sci., 112, 305-319.

,-- , and Taylor, J. (1963). Alterations of visual evoked response in the presence of homonymous visual defects. Electroenceph. clin. Neurophysiol., 15, 737-746. 